\title{
Relativistic attosecond electron bunch emission from few-cycle laser irradiated nanoscale droplets
}

\author{
Laura Di Lucchio ${ }^{1}$ and Paul Gibbon ${ }^{1,2}$ \\ ${ }^{1}$ Forschungszentrum Jülich GmbH, Institute for Advanced Simulation, Jülich Supercomputing Centre, \\ D-52425 Jülich, Germany \\ ${ }^{2}$ Centre for Mathematical Plasma Astrophysics, Department of Mathematics, KU Leuven, \\ Celestijnenlaan 200B, 3001 Heverlee, Belgium
}

(Received 1 August 2014; published 26 February 2015)

\begin{abstract}
Attosecond electron bunches produced at the surface of nanometer-scale droplets illuminated by a two-cycle laser pulse are investigated for the purpose of determining their optimal emission characteristics. Significant departures from Mie theory are found for electron bunch emission from droplets whose radii satisfy the condition $\delta_{r}<R<10 \delta_{r}$, where $\delta_{r}=\gamma^{1 / 2} c / \omega_{p}$ is the plasma relativistic skin depth; an effect which can be accounted for by induced transparency. Scattering from such droplets is subject to a transitional regime which is neither accounted for by optical Mie theory valid for $R \gg \delta$, where $\delta$ is the usual plasma skin depth, nor with the Rayleigh regime $(R<\delta \ll \lambda)$. Instead the angular emission of the bunches is to a good approximation described by the nonlinear ponderomotive scattering model. Subsequently, the bunches are subject to further deflection by the ponderomotive pressure of the copropagating laser field in vacuum, depending on the initial droplet parameters. Final emission angles are estimated, together with the energy spectrum of the bunches.
\end{abstract}

DOI: 10.1103/PhysRevSTAB.18.023402

PACS numbers: 41.75.Jv, 52.38.Kd, 52.59.Bi, 52.59.Fn

\section{INTRODUCTION}

Ultrashort electron bunches created by the interaction of high-intensity, femtosecond laser pulses with plasma targets have received much interest recently because of their potential as a powerful source of attosecond X-ray pulses. The latter can be produced in X-ray tube fashion by bombarding a secondary target with the electron bunches, and have an immediate application in ultrafast X-ray microscopy and atomic dynamics studies. Several mechanisms have already been proposed for producing collimated attosecond electron bunches, such as vacuum acceleration by tailored laser pulses [1], interaction of p-polarized laser pulses with overdense plasma boundaries [2], stochastic slicing of electron pulses [3], interaction of laser pulses with nanofilms [4], inverse free-electron laser interactions [5], laser-illuminated droplets [6], laser illuminated ultrathin plasma layers [7] and attosecond electron sheets from plasma wakefields $[8,9]$. Among these various techniques, masslimited droplet targets may offer a number of advantages in terms of the electron bunch properties such as high density and low emittance. The ionization and heating mechanisms in laser-illuminated rare gas clusters or droplets have been thoroughly investigated in recent years, as summarized in Refs. [10-12]. Other work with clusters has concentrated on the ion dynamics in cases for which the size and density of the

Published by the American Physical Society under the terms of the Creative Commons Attribution 3.0 License. Further distribution of this work must maintain attribution to the author(s) and the published article's title, journal citation, and DOI. cluster give rise to complete ionization, resulting in a Coulomb explosion and high proton energies [13]. A general review on laser cluster interaction dynamics is presented in Ref. [14]. Quite recently, nonlinear optical behavior induced by the interaction of micron-sized clusters with strong nearinfrared or optical laser pulses has been analyzed [15], primarily in the nonrelativistic regime, i.e., laser intensities below $10^{18} \mathrm{~W} / \mathrm{cm}^{2}$.

Despite this wealth of prior work, little attention has so far been paid to the dynamical behavior of droplets whose radius is small compared to the incident wavelength, i.e., a few hundreds or even tens of nanometers, illuminated by laser light at intensities $I>10^{19} \mathrm{~W} / \mathrm{cm}^{2}$. However, such a physical scenario is now accessible at a number of laser laboratories [16], and is therefore a favorable target configuration for producing energetic electron bunches. A previous investigation on electron bunch generation that serves as a useful baseline for the present work is that by Liseykina et al., [6] who focused their attention on lowdensity (Helium) droplets interacting with a 16-cycle laser pulse. State-of-the-art, few-cycle (5 fs) pulses at relativistic intensities allow analogous but potentially cleaner conditions for investigating the cluster interaction dynamics. Recent experimental work [17] has demonstrated attosecond control of the collective electron motion and directional emission from isolated dielectric $\mathrm{SiO}_{2}$ nanoparticles with phasestabilized two-cycle laser pulses. So far such experiments have been performed at relatively low field intensities, where the response of the medium is still linear; however, free nanoparticles have been discovered to be particularly advantageous for studying the nonlinear regime in very strong fields, such as those generated by relativistic laser pulses. 
Ultrashort pulses have the particularity of allowing the measurement of electron dynamics without any significant influence by the motion of the nuclei during the laser time scale, thus allowing investigation of novel highly nonlinear regimes for the instantaneous response of the solid medium to ultraintense illumination. The remainder of this paper is structured as follows: first we describe the numerical model used for the investigation, illustrating some of the typical characteristics of electron bunch emission in the few-cycle, high-intensity regime. Next, we summarize the main findings concerning the emission angle and proceed to discuss these results in terms of available theories. Finally we conclude with an outlook on near-term experimental work.

\section{SIMULATION DETAILS}

To begin with we demonstrate efficient generation of ultrashort dense electron bunches at the surface of a small droplet illuminated by a two-cycle (fwhm $=5 \mathrm{fs}$ ) ultraintense Gaussian laser pulse; in contrast to Ref. [6] our spherical nanotargets have a solid-like density of $n=100 n_{c}$, where $n_{c}=1.8 \times 10^{21} \mathrm{~cm}^{-3}$ is the critical density for $800 \mathrm{~nm}$ wavelength light. Clusters with a range of sizes have been considered, starting with a much smaller radius than the laser focus, namely of $100 \mathrm{~nm}$, comparing their dynamics with clusters up to a 1 micron radius, that is, a size comparable to the focus. The intensity of the laser was varied between $10^{17}-10^{21} \mathrm{Wcm}^{-2}$ to determine scaling behavior for the emitted particles' energies and emission angles. The focus of the Gaussian pulse has been kept fixed to 1 micron, after verifying that the interaction dynamics does not change for variations up to 3 microns. Our simulations have been performed with the particle-in-cell code EPOCH [18], mostly in two dimensions with a few three-dimensional runs for comparison. The simulation box size was varied from 4-20 $\mu \mathrm{m}$, depending on cluster size and intensity, while the cell size was kept fixed at $\approx 8 \mathrm{~nm}$. The number of simulation particles was chosen to maintain sufficient statistics for different cluster sizes, ranging from $34 \times 10^{6}$ (equally subdivided among ions and electrons) for the $100 \mathrm{~nm}$ droplet, to $34 \times 10^{7}$ for the 1 micron droplet.

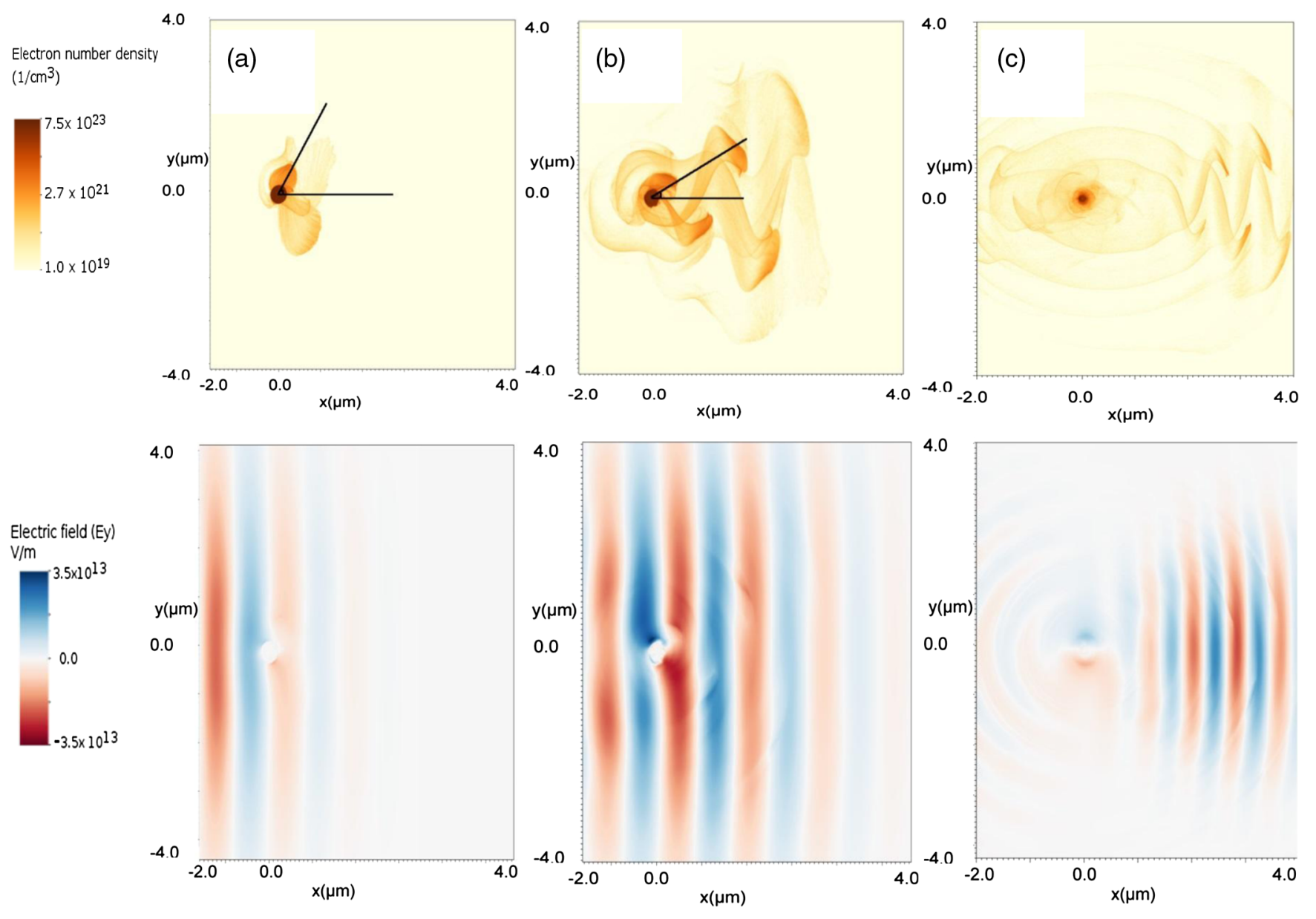

FIG. 1. Laser electric field (bottom, in $\mathrm{V} / \mathrm{m}$ ), and electron number density (top, in $\mathrm{m}^{-3}$ ) plot for a $100 \mathrm{~nm}$ radius droplet hit by a laser pulse of intensity $I=10^{20} \mathrm{~W} / \mathrm{cm}^{2}$ at times $\approx 3 \mathrm{fs}(\mathrm{a}), 8 \mathrm{fs}(\mathrm{b}), 16 \mathrm{fs}(\mathrm{c})$. The cluster is centered at position $(0,0)$. Angles are measured relative to the center of the most dense region of the bunch. The logarithmic density scale is normalized to the critical density $n_{c}=1.8 \times 10^{21} \mathrm{~cm}^{-3}$, so that the maximum value corresponds to $100 n_{c}$. The maximum absolute value of the electric field is $3.5 \times 10^{13} \mathrm{~V} / \mathrm{m}$. 
The dynamics of the electrons expelled from the droplet proves to be quite complex: early emission occurs after the first half-cycle of the pulse, the emitted bunches are then deflected forming several optically thin cusps at different inclinations at the rear side of the cluster, depending on droplet size and laser intensity. The electrons appear to interact with the pulse twice, first when they are still near the droplet surface and later during escape in vacuum. A typical example of this phenomenon is shown in Fig. 1. It is also noticeable how the laser field itself is perturbed by the co-propagating emitted bunches, which sit close to the field null-points.

\section{RESULTS}

We now proceed to investigate the emission angle distribution as a function of the laser intensity and droplet size, in order to predict when and where the most intense bunches might be detected experimentally. On the $10 \mathrm{fs}$ interaction time scale relevant to the few-cycle laser drivers considered here, the dynamics of the electrons and ions are well separated: as far as the electron bunch generation is concerned, the ion dynamics can be ignored. The electrons start to move as soon as the leading edge of the laser pulse reaches the droplet; their subsequent dynamics depends on the droplet size and the laser intensity. Two physical processes can in principle contribute to the final angular emission of electron bunches: (i) Mie scattering theory $[19,20]$ and (ii) ponderomotive scattering [21]. It turns out that there is a transition between these two effects for particular combinations of the laser and target parameters.

In fact our numerical results already show departures from Mie scattering patterns as in [6] for droplets whose radius is lower than $\lambda / 2$ at relativistic laser intensities. Electrons are emitted along two alternating directions corresponding to the same optimal angle but different orientation within the polarization plane (of the laser electric field), with respect to the laser propagation axis, $\left(0^{\circ}\right)$; these angles depend also on the intensity rather than only the droplet size, in contrast to the linear Mie regime

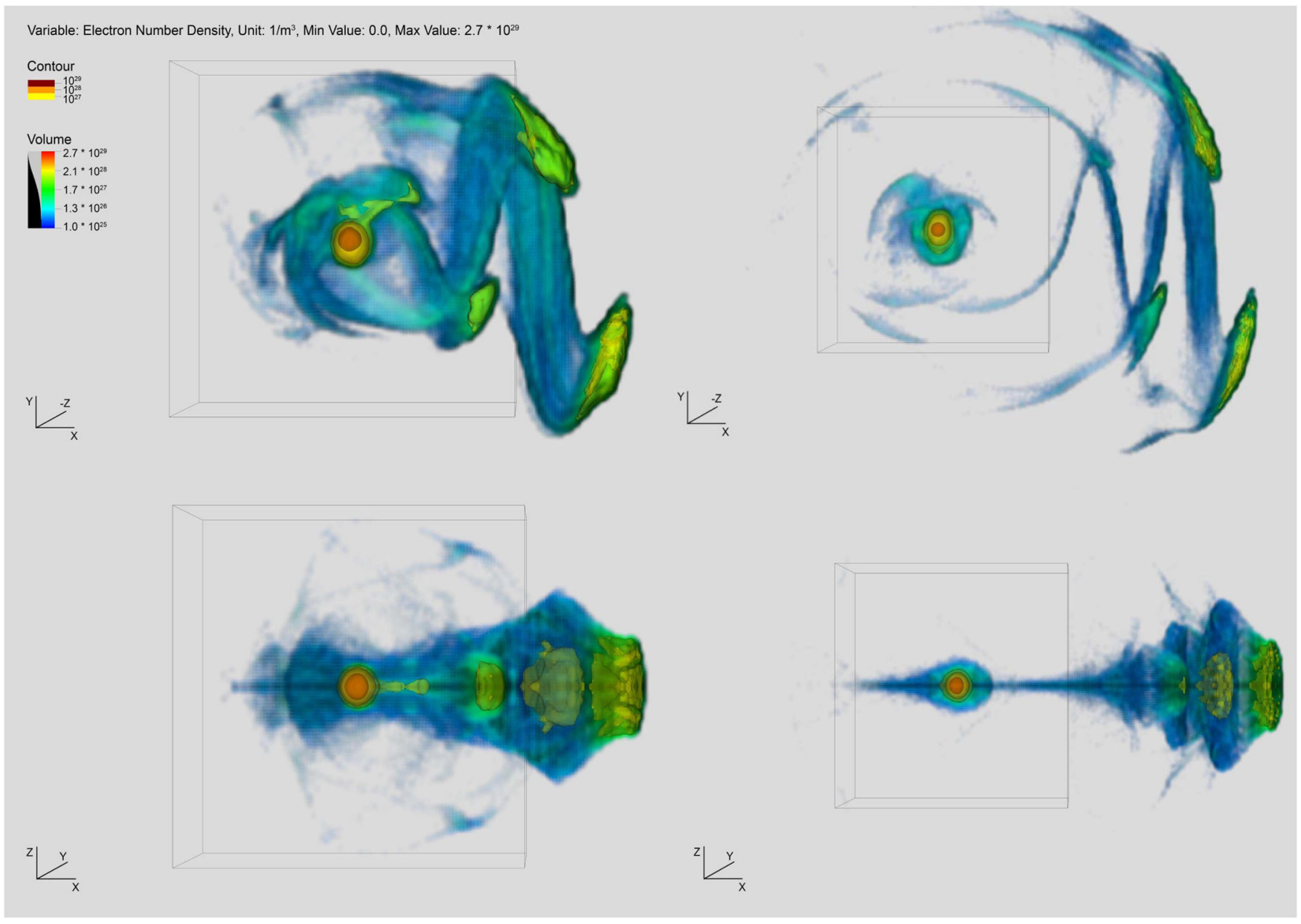

FIG. 2. Logarithmic plot of the electron number density for a 100 times overdense droplet with $R=100 \mathrm{~nm}$ as it evolves in the field of a linearly polarized Gaussian laser pulse with $I=10^{20} \mathrm{~W} / \mathrm{cm}^{2}$. The contoured regions represent the dense regions which can be identified as the proper nanobunches, at, respectively, $t=10 \mathrm{fs}$ (left) and $15 \mathrm{fs}$ (right) after the start of the laser-droplet interaction. Two main perspectives are represented: xy-plane view (up) and xz-plane (down). 


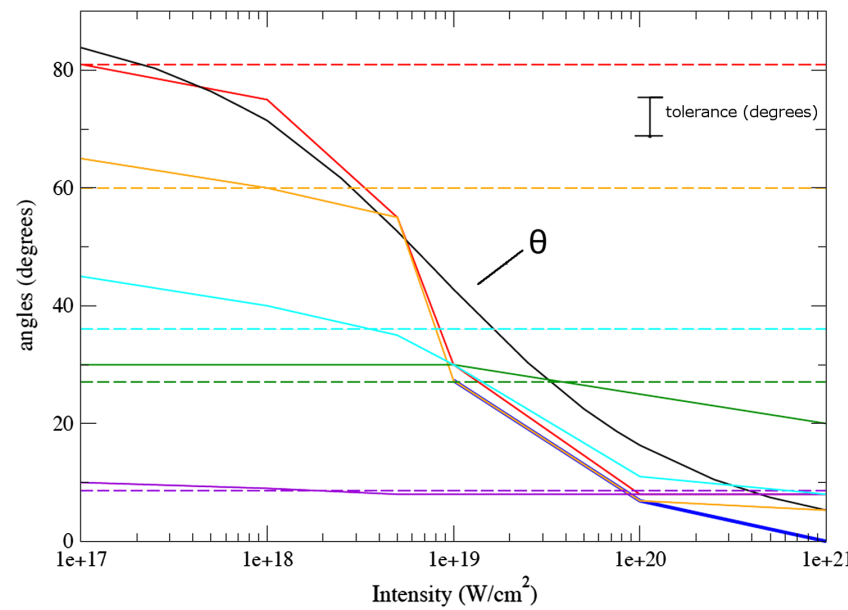

FIG. 3. Electron emission angles as a function of the laser intensities, for different droplets sizes, compared with available models. Color corresponds to different cluster radii, namely: red $=100 \mathrm{~nm}, \quad$ orange $=200 \mathrm{~nm}, \quad$ cyan $=300 \mathrm{~nm}, \quad$ green $=$ $500 \mathrm{~nm}$, violet $=1$ micron. Solid lines represent simulation points, dashed horizontal lines Mie theory prediction. Black and blue line represent nonlinear ponderomotive scattering prediction and deviation formula of Eq. (3), respectively. The observed divergence with respect to central direction of the bunches is about 5 degrees.

described later in Sec. IVA. The angular emission for different representative droplet sizes as a function of the intensity is summarized in Fig. 3; angles inferred from simulations are measured while the laser is passing through the droplet, i.e., on the attosecond time scale. Two limiting cases are immediately apparent from Fig. 3: the low intensity, large droplet radius case (bottom left), and the high intensity/small radius limit where the simulations follow the ponderomotive scattering behavior. In order to verify that the electron bunch emission occurs within the laser wave polarization plane, we have investigated the early laser-droplet dynamics by repeating some of the simulations in $3 \mathrm{D}$. In order to maintain the same accuracy at manageable computational cost, a reduced box of $6 \times 4 \times 4 \mu \mathrm{m}$ has been chosen, in order to fully represent in detail the first $20 \mathrm{fs}$ of electron motion. The evolution of electron number density shows the initial emission of the bunches in a spherical geometry, and their subsequent alignment in the polarization plane together with the outgoing wave (see Fig. 2), thus confirming our assumption that the final directions of propagation can be studied and determined by means of 2D simulations only.

\section{DISCUSSION}

In the following we discuss the results of Fig. 3 in detail, and attempt to explain the differences between the emission angles observed in the simulations and the behavior expected in the linear (Mie) and relativistic regimes, respectively.

\section{A. Emission angles according to Mie theory}

The linear optical or Mie-regime of electromagnetic wave scattering from a spherical conductor is characterized by the existence of a field enhancement pattern at different orientations on the surface. In order to fully compare our simulations with this regime, the scattered spatiotemporal surface field as predicted by Mie theory for several droplet sizes is presented in Fig. 4. In our Mie calculation, which uses the program of Ref. [20] the droplet is conducting with dielectric constant $\epsilon=1-\omega_{p}^{2} / \omega^{2}$, and the incident plane wave field is $E_{\text {inc }}(z, t)=E_{0} \cos (k z-\omega t)$ with a constant amplitude. From Fig. 4 it is clear that for the smaller droplets the angle of maximum field enhancement approaches $\pi / 2$, whereas for bigger droplets the first maximum is closer to the laser pulse axis $(\theta=0)$, and secondary maxima also appear.

The field enhancement in this direction seen is up to a factor of 4 , somewhat higher than the maximum radial field at the surface predicted by theory in the small cluster limit. In the latter case $k R=2 \pi R / \lambda \ll 1$ (where $R$ is the radius of the droplet), and the following formula holds:

$$
E_{r}^{\max }=3 E_{0} \frac{\omega_{p}^{2} / \omega^{2}-1}{\omega_{p}^{2} / \omega^{2}-3}, \quad k R=2 \pi R / \lambda \ll 1,
$$

which would yield $E_{r}^{\max } \approx 3 E_{0}$ in the high density limit $\omega_{p}^{2} \gg \omega^{2}$. The deviation is reasonable considering that in our case $k R \approx 0.79$. However, in the simulation output the enhancement of the electric field is lower and transient; it can hardly be inferred from electric field images and detailed vectorial calculations both in $2 \mathrm{D}$ and in $3 \mathrm{D}$ yield a factor ranging from 2 for the smallest droplets to just slightly above 1 for the bigger ones. This could be due to the fact that the above calculations do not include inwardscattered waves which could weaken the field at the surface. Another issue could be related to the construction of the dielectric in the PIC simulations; the charge is calculated at grid points and assembled during the field evolution, so that a dielectric constant is no longer a good approximation. Mie theory is based on the assumption that the condition $R \gg \delta$ is satisfied, where $\delta=c / \omega_{p}$ is the plasma skin depth. It also neglects ionization, which also could produce a temporal variation in the dielectric constant. As a consequence, the refraction index of the medium is not maintained; however, we consider this effect as of secondary importance when high laser intensities are reached and do not include it in our simulations. Therefore we can assume a constant $\epsilon$ for all cases in spite of the intrinsic limitations in particle-in-cell calculations. A general result is found, as clearly manifest from Fig. 3, that in interactions with smaller droplets $(R=100-200 \mathrm{~nm})$ the observed emission direction diverges from the constant angle predicted by Mie theory as soon as the laser intensity becomes relativistic. 

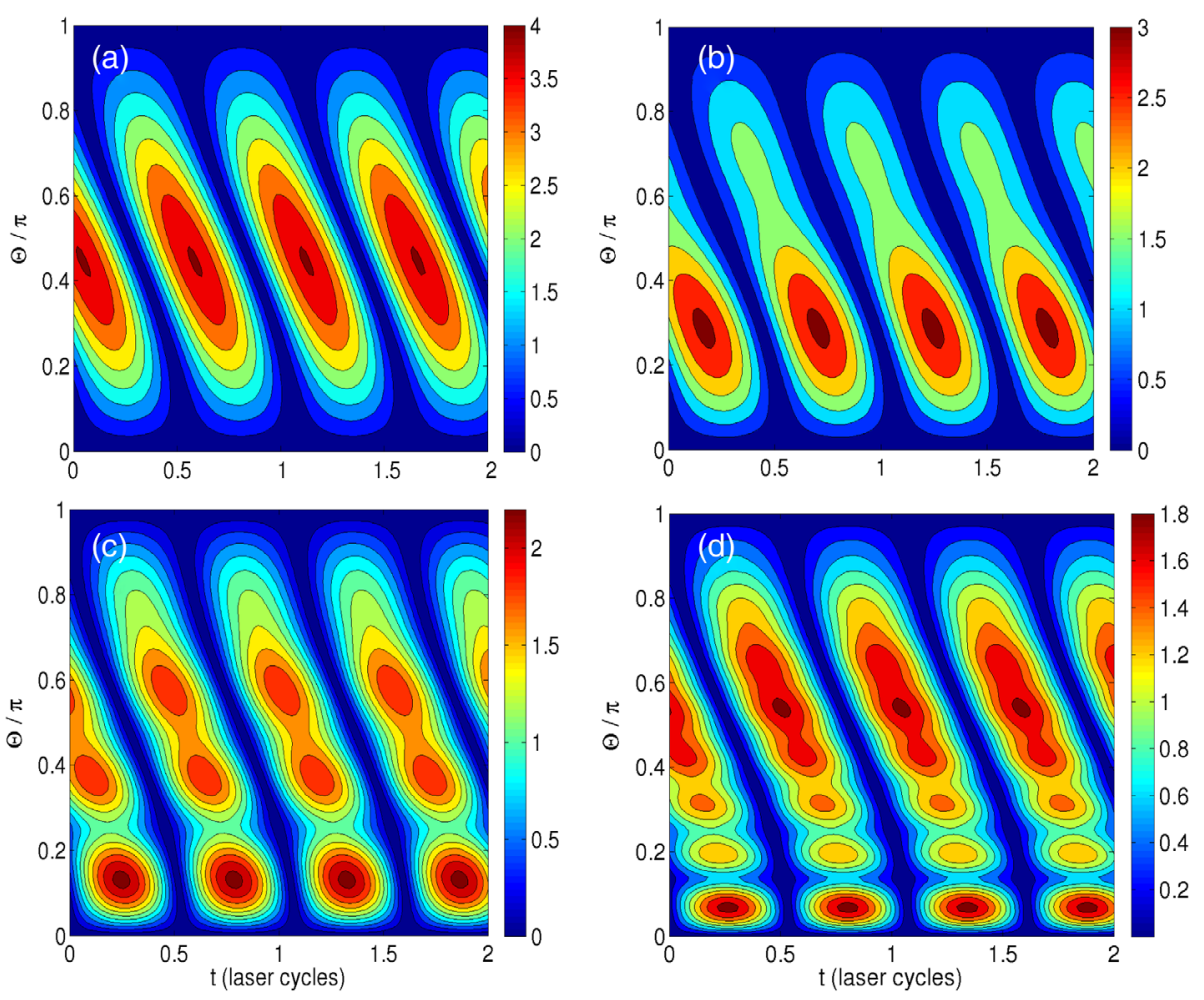

FIG. 4. Absolute value of the radial electric field for a 100 times overdense droplet with $R=100 \mathrm{~nm}(\mathrm{a}), 200 \mathrm{~nm}$ (b), $500 \mathrm{~nm}(\mathrm{c})$, and 1 micron (d) in an incident plane wave of wavelength $\lambda=800 \mathrm{~nm}$ as a function of time and angle on the surface. The color scale indicates the enhancement factor of the incident electric field $E_{0}$, for a constant plane wave amplitude.

\section{B. Relativistic regime: Ponderomotive scattering}

The discrepancy between the predicted Mie angles of Fig. 4 and the actual emission angles of the electrons clearly has its origin in some other physical mechanism during the ejection of the electron bunches. A few remarks can be made here regarding this issue. First of all, in our simulations we are dealing with an ultrashort tightly focused Gaussian laser pulse, which cannot be straightforwardly approximated by an infinite plane wave of constant amplitude as assumed in Mie scattering. Mie theory constructs a finite numerical expansion of the constant plane wave in terms of spherical vector wave functions in order to calculate the scattered and absorbed radiation, which may not be valid for a finite-size modulated waveform. Furthermore, in our cases the laser intensity is relativistic, whereas the linear Mie theory is only valid for small field amplitudes, i.e., $e E_{L} / m \omega c \ll 1$.

On the other hand, the observed transition only occurs for droplets with $R<\lambda / 2$ which means that the transition is both size- and intensity-dependent. In order to explain this, we need to reexamine the usual condition for the validity of the Mie optical regime, namely that the radius of the droplet is far greater than the plasma skin depth. This is necessary because Mie theory assumes that the electromagnetic fields inside and outside the droplet undergo a smooth transition at a sharp, infinitely thin boundary between the droplet and its surroundings. When the size of the droplet is less than its skin depth the fields see it as a surface rather than a bulk medium, and the laser pulse is not completely screened from the interior, thus being transmitted rather than reflected. The scattered field then has little influence on the dynamics of the emitted electrons.

Our simulation results for 100-200 nm droplets are not in agreement with any of these models but the parameters suggest that this represents a transition regime where $R \sim \delta_{r}$; where $\delta_{r}=\sqrt{\gamma} \delta$, the larger effective skin depth resulting from relativistically induced transparency. [22] Here the effective plasma frequency is $\omega_{p}^{r}=\omega_{p} / \sqrt{\gamma}$ and therefore for $\gamma=\sqrt{1+a_{0}^{2}} \approx 5-20$, we have $\delta_{r} \approx 40-60 \mathrm{~nm}$, which is of the same order as the radius $R$ for the smaller droplets. In this regime it makes more sense to use a relativistic interaction model such as the ponderomotive scattering of electrons by an intense field in the laser focus as treated, for example, in [21], and first demonstrated by Meyerhofer [23] and Malka [24].

Following the study in Ref. [21], the scattering angle of a single electron in the laser focus in vacuum is given by

$$
\theta_{0}=\arctan \frac{\sqrt{\frac{2}{1+\beta_{0}}\left(\frac{\gamma}{\gamma_{0}}-1\right)}}{\gamma-\gamma_{0}\left(1-\beta_{0}\right)}
$$

where $\gamma, \gamma_{0}$, and $\beta_{0}$ are, respectively, the final energy, the initial energy and the normalized parallel velocity of the 
particle: for an electron initially at the rest on the droplet surface, $\gamma_{0}=1$ and $\beta_{0}=0$, in which case Eq. (2) reduces to $\theta_{0}=\arctan \sqrt{2 /(\gamma-1)}$, the black curve drawn in Fig. 3 .

\section{Electrostatic deflection}

Although the ponderomotive model reproduces the general trend of the simulation results, there is still some additional deflection not accounted for at higher intensities. During the motion of the bunches the fronts appear to be bent by the differential action of radiation pressure force on higher and lower density regions: the latter have a smaller reflection index and feel a smaller pressure thus lagging behind the laser pulse in time. The ponderomotive pressure induces oscillations in the momenta, whose amplitude is lower in the most dense regions due to the fact that they feel a greater restoring force. Such dense regions are overcritical $\left(n_{b}>n_{c r}\right)$ for relativistic laser intensities and can be identified as proper bunches due to their narrowness. They can be described in terms of two parameters, namely the number of particles $N_{b}$ they contain and their longitudinal thickness $d_{b}$. In Fig. 5 a one-dimensional lineout of the electron density along the propagation direction of the bunches has been drawn for a $100 \mathrm{~nm}$ droplet hit by a laser pulse of intensity $I=10^{20} \mathrm{~W} / \mathrm{cm}^{2}$ at a time of $16 \mathrm{fs}$ after the interaction. The four bunches correspond to those in Fig. 1c and are aligned along the orientation predicted by Eq. (2). Furthermore, their spatial extension is about $d_{b}=100 \mathrm{~nm}$.

As long as the electrons kinetic energy overcomes the electrostatic potential energy of the bunch (Coulomb barrier threshold), that is, $N_{b} \epsilon_{e}>e^{2} N_{b}^{2} / d_{b}$, [25] the normal component of the momentum is progressively reduced by an amount corresponding to the space-charge

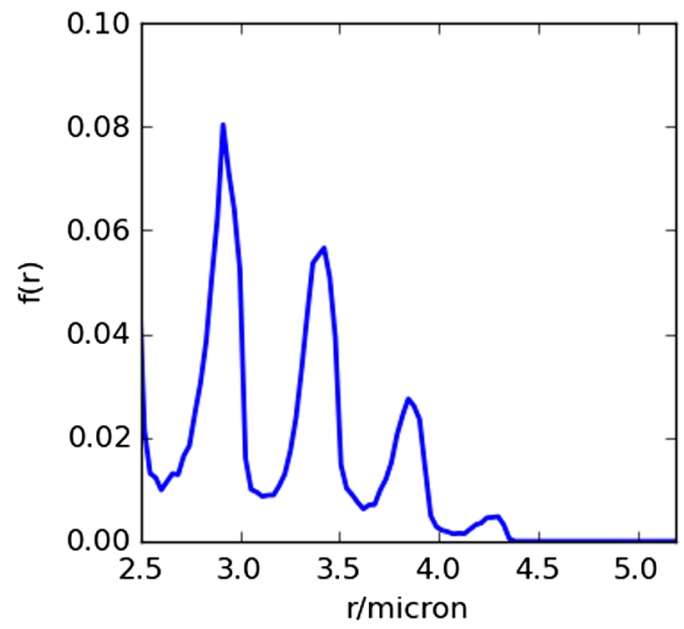

FIG. 5. Number of particles $f(r)$ as a function of the radial distance $r$ from the center of the droplet, integrated over all angles for a droplet with radius $R=100 \mathrm{~nm}$ illuminated by a laser at intensity $I=10^{20} \mathrm{~W} / \mathrm{cm}^{2}$. The particles are probed at a time corresponding to Fig. 1c. force contribution from the ionized droplet. This causes a further lowering of the angle of propagation of the dense bunches. Such an evolution takes place as soon as the laser intensity is relativistic: for a $100 \mathrm{~nm}$ droplet and a laser intensity $I=10^{19} \mathrm{Wcm}^{2}$ one can estimate $N_{b} \approx 10^{8}$ and $d_{b} \approx 100 \mathrm{~nm}$ from the simulations, and the corresponding threshold energy of $1.4 \mathrm{MeV}$ is easily overcome. Such a spatial extension of the bunches corresponds to a duration of 300 attoseconds and a total charge yield around $10 \mathrm{pC}$, or a current of $33 \mathrm{kA}$; for higher intensities, a few tens of $\mathrm{pC}$ can be reached, at least when the bunches are ejected from the surface of the droplet, as discussed later in Sec. IV E. Defining $p_{\perp}$ and $p_{\|}$as the components of the momentum along the perpendicular and tangential directions with respect to the droplet surface, the emission angle $\theta=$ $\arctan \left(p_{y} / p_{x}\right)$ changes from the initial scattering angle $\theta_{0}$ [see Eq. (2)], due to a lowering of the absolute value of $p_{\perp}$, in a symmetrical way as described for [25] in the case of bunches copropagating at some angle together with a reflected pulse. Making the equations explicit we have (see Fig. 6 for geometrical visualization):

$$
\begin{aligned}
\theta & =\arctan \left(\frac{p_{y}}{p_{x}}\right)=\theta_{0} \\
p & =\frac{p_{x}}{\cos \theta}=\frac{p_{y}}{\sin \theta}=\frac{1}{c} \epsilon_{e} \\
p_{\perp} & =p_{y} \sin \theta \\
p_{\|} & =p_{x} \sin \theta \\
p_{\perp}^{\prime} & \approx p_{\perp}-e^{2} N_{b m} / c d_{m} \\
p_{\perp}^{\prime} & =p_{y}^{\prime} \sin \theta^{\prime} \\
p_{\|}^{\prime} & =p_{x}^{\prime} \sin \theta^{\prime}=p_{\|} \\
\theta^{\prime} & =\arctan \frac{p_{y}^{\prime}}{p_{x}^{\prime}}+\arctan \frac{p_{\perp}}{p_{\|}}
\end{aligned}
$$

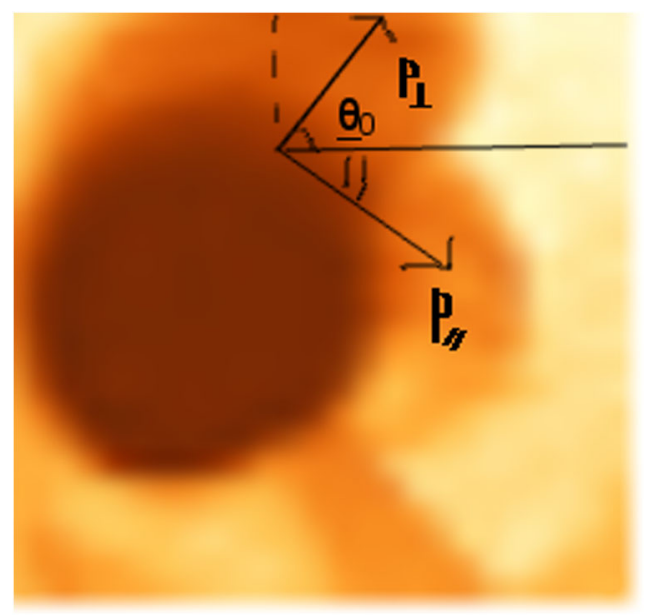

FIG. 6. Geometry of bunch deflection due to space charge. 
Equations (3) are only valid in the range of intensities $10^{19}-10^{21} \mathrm{~W} / \mathrm{cm}^{2}$, with the Coulomb explosion regime presenting the peculiarity of an emission concentrated along the laser pulse axis, and the angular deviations are shown in the corresponding curves in Fig. 3. An example of this additional deviation for a laser intensity $I=$ $10^{20} \mathrm{~W} / \mathrm{cm}^{2}$ and a droplet of $100 \mathrm{~nm}$ radius is displayed in the montage plot Fig. 1 of the electron density evolution at times corresponding to the initial Mie and ponderomotive emissions, followed by the further deviation of the bunches in vacuum. The predicted deviation for an electron in a bunch emitted at $17^{\circ}$ is a lowering of its inclination down to $\theta^{\prime} \approx 6.9^{\circ}$, in good agreement with the direction of the most dense bunches fronts observed at $t \approx 20$ fs after the interaction.

\section{Post-propagation effects}

Once the bunches have left the droplet they start traveling in the field of the scattered laser pulse, analogously to what happens when a short laser pulse is reflected from a finite-sized plane target as described in Ref. [25]. In our case, however, most of the pulse is actually transmitted rather than reflected, so that the highest field gradients are in the rear side vacuum region, where also most of the electrons are expelled-Fig. 7.
The evolution of the motion of the bunches while they are co-propagating with the laser wave is illustrated in Fig. 7, where the electric field energy density and the electron number density have been superposed on the same plot, showing the spatial coincidence between the field maxima and the regions where the electron bunches are actually sitting. This happens for all sizes, however, for the bigger droplets (i.e., 1 micron) the reflected field shows an intensity comparable with the incident and scattered ones, even if only at later times, after the electron bunches have already well escaped in vacuum. During the motion, the nanobunches spread out along the emission direction predicted in Fig. 3, which is however maintained as their axis direction. One can easily infer that this will happen also at even later times, for example when the bunches hit a detector at a time of the order of seconds after the start of the interaction. The peak in the signal generated by the bunches should then be still in the predicted spatial orientations, but there could be changes in the magnitude of the emittance which cannot be quantitatively determined by means of the present simulations.

An upper limit to the copropagation time, neglecting 3D diffraction effects, can be estimated as follows: if $\tau_{l}$ is the laser pulse duration, the co-motion of the laser wave and the electrons lasts for a period $t=c \tau_{L} /(c-v) \approx$ $\tau_{L}\left(\epsilon_{e} / m_{e} c^{2}\right)^{2}$, where $\epsilon_{e}$ is the mean electron kinetic
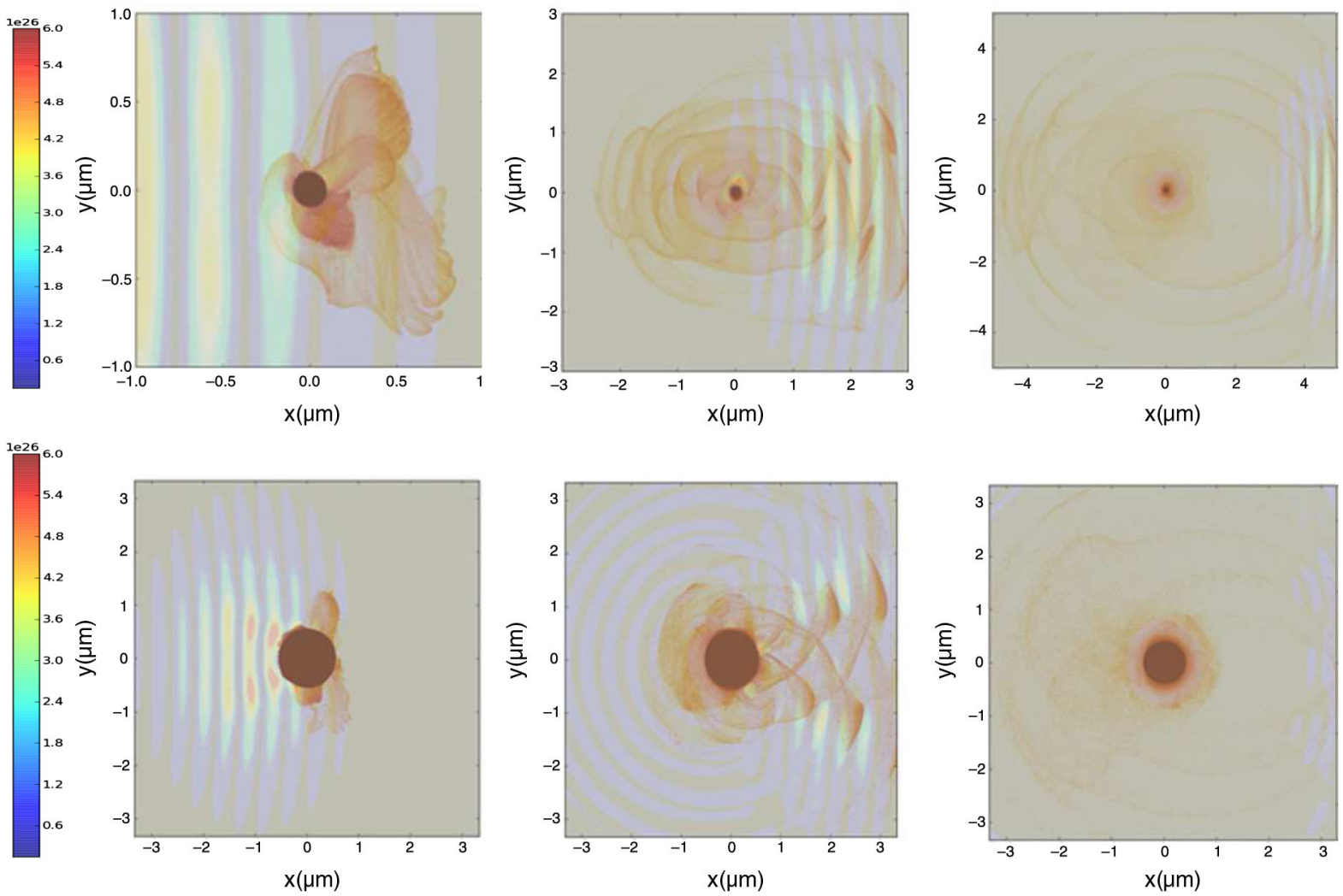

FIG. 7. Electric field energy density (in legend), superposed with the logarithmic plot of number electron density (in units of critical density $1.8 \times 10^{27} \mathrm{~m}^{-3}$ ) for a droplet with radius $100 \mathrm{~nm}$ (above) and 1 micron (below) respectively hit by a laser pulse of intensity $I=10^{20} \mathrm{~W} / \mathrm{cm}^{2}$ at times $\approx 3 \mathrm{fs}(\mathrm{a}), 8 \mathrm{fs}(\mathrm{b}), 16 \mathrm{fs}(\mathrm{c})$. Angles are measured considering the center of the most dense region of the bunch. In the logarithmic density scale, the maximum value corresponds to the critical density (see Fig. 1). 
energy. For $I=10^{20} \mathrm{Wcm}^{-2}$ and an average energy of $\epsilon_{e} \approx 7 \mathrm{MeV}$ this period is equal to $\approx 1 \mathrm{ps}$, equivalent to $0.3 \mathrm{~mm}$ travel distance. This assumes that the bunches' trajectories remain parallel to the EM wavefronts, which may not be the case after allowing for diffractionespecially for tight laser foci-an effect which would shorten the copropagation time. Diffraction also reduces the intensity of the local laser field experienced by a comoving bunch and may lead to further longitudinal spreading over many Rayleigh lengths. In our simulations, the bunches have already acquired a stable form at one Rayleigh length, an observation confirmed in additional simulations for the $100 \mathrm{~nm}$ radius droplet and $10^{20} \mathrm{Wcm}^{-2}$ laser intensity for different focus sizes up to 3 microns. In these cases no significant attenuation effects up to a distance of 6 micron from the droplet surface were found. However, a complete treatment of the full propagation of the bunches into the far field, i.e., distances of the order of $\mathrm{cm}$, is beyond the scope of this work. In general a detector signal is likely to be time-integrated, and may not be able to resolve spreading of the particles around the propagation direction due to emittance.
All droplets with $R<\lambda / 2$ follow a similar evolution, with the quantitative angular differences in angles stated by Eqs. (2) and (3). The final angular energy spectra for three relevant values of laser intensity, together with the spectra of the single bunches, are shown in Fig. 8. According to the calculations for the outrunning time of an electron in the bunch with respect to the laser pulse, the stabilization on the final angle of emission takes a time which increases with the laser intensity; however, a representative picture can still be obtained at about $20 \mathrm{fs}$ after the interaction. The highest intensity case is a peculiar one, since for the corresponding parameters Eq. (3) give a negative final angle, whose meaning could be interpreted as a leakage of the bunch toward the opposite direction constituting a further contribution to the electron emission across the $\mathrm{x}$-axis. However, this cannot be directly inferred from simulations as the computational means required to follow the bunches until they decouple from the laser pulse-that is, for hundreds of femtoseconds or picoseconds are beyond the reach of this study. The energy spectra pictures confirm that the energetic electron emission remains close to the
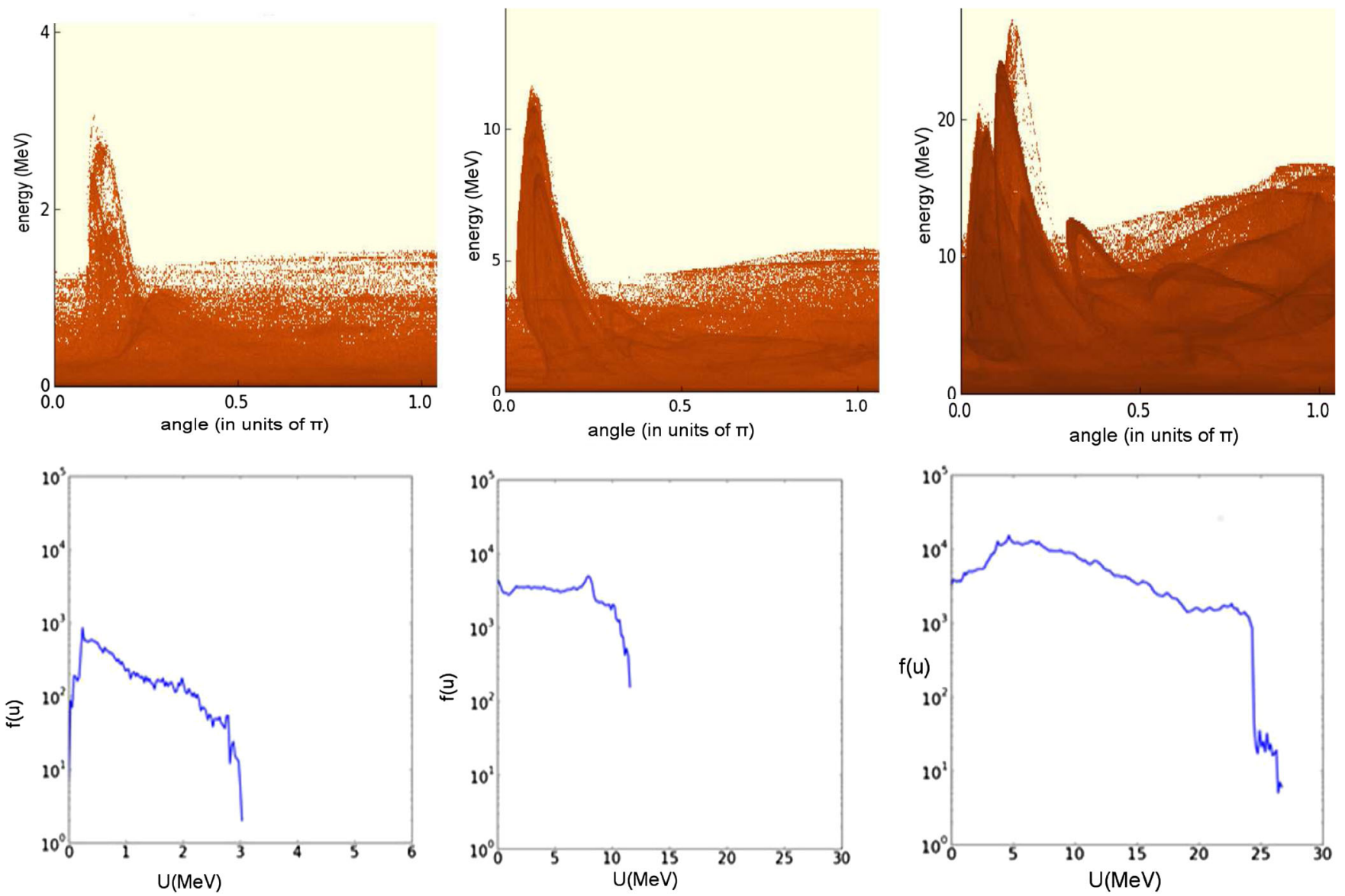

FIG. 8. Angular energy distributions (above) and single bunch energy spectra (below) at $t \approx 20$ fs after the start of the interaction, for a $100 \mathrm{~nm}$ radius droplet hit by a laser pulse of intensities $I=10^{19} \mathrm{~W} / \mathrm{cm}^{2}, I=10^{20} \mathrm{~W} / \mathrm{cm}^{2}$, and $I=10^{21} \mathrm{~W} / \mathrm{cm}^{2}$. 
$\mathrm{x}$-axis (Fig. 8): the spectra of the bunches are characterized by an increasing spread in energies at higher intensity.

\section{E. Dependence on droplet size}

For higher droplet sizes, i.e., $\lambda / 2<R \leq \lambda$, the approximation $R \gg \delta=c / \omega_{p}$ is always valid regardless of induced transparency effects, and Mie emission is important for all intensities studied, causing the appearance of one or more secondary emission angles for energetic electrons, as predicted by the patterns in Fig. 4. The $400 \mathrm{~nm}$ droplet represents a transition size, where Mie and scattering predictions are very close to each other in this case. For a $500 \mathrm{~nm}$ droplet, the dynamics is clearly different with respect to the smaller sizes: the laser pulse is strongly diffracted and diffused, both outwards and inwards; less than a quarter of the wave energy is propagating outward after hitting the cluster. The electric field and density plots for subsequent evolution times are shown in Fig. 9, which shows a threefold early emission of underdense bunches $\left(n_{b}<n_{c r}\right)$ in agreement with Mie predictions as in Fig. 4. The final electron energies are below $1 \mathrm{MeV}$, therefore insufficient for the overcoming of the Coulomb barrier; emission fronts are not further bent while passing through the outcoming pulse, therefore the angular spread is much higher than in the smaller droplet case (up to $\pm 10^{\circ}$ ). The final emission shows therefore a wide distribution around Mie orientations. The extreme case of a 1 micron droplet $(R \approx \lambda)$ exhibits the same behavior, except for a complicated angular distribution due to the presence of many secondary maxima for field enhancement at the surface as predicted in Fig. 4. In order to better compare this limiting situation with emission from smaller droplets, the number of emitted particles per bunch has been calculated and it was found to be two orders of magnitude smaller than the corresponding one in the $100 \mathrm{~nm}$ droplet case; these low density bunches only yield a total charge of a few fractions of $\mathrm{pC}$, or a few $\mathrm{pC}$ for very high intensities $\left(I_{L}>10^{21} \mathrm{~W} / \mathrm{cm}^{2}\right)$.

The charge contained in the emitted bunches for small and large droplet sizes is summarized in Table I. Comparisons with other schemes for attosecond electron bunch generation indicate that laser-illuminated nanometer-scale droplets are indeed competitive as a source, yielding single bunch charges well above the $\mathrm{pC}$ level. The post-propagation of the bunches with the laser
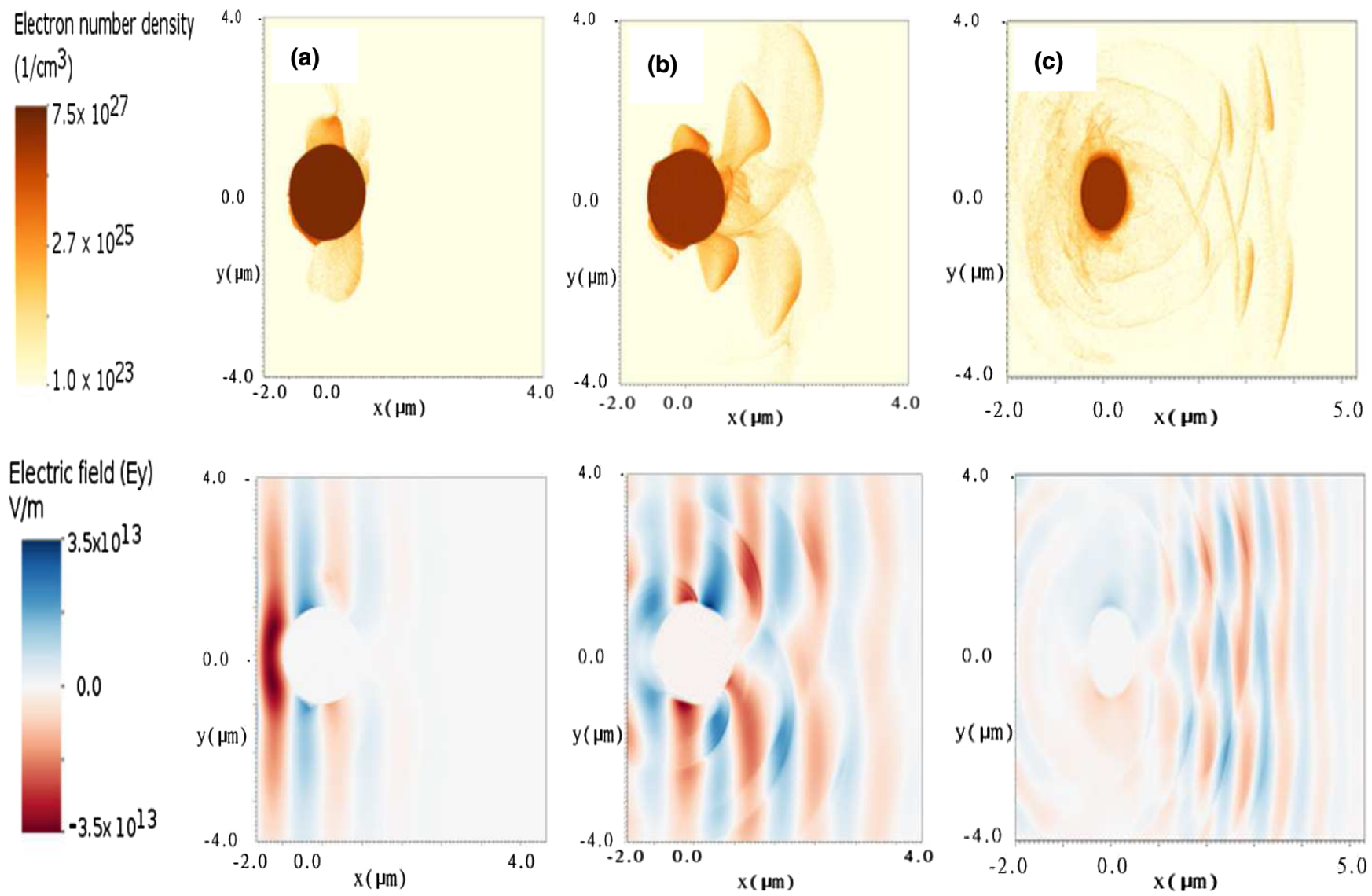

FIG. 9. Electric transverse field (in $\mathrm{V} / \mathrm{m}$ ), and electron density (in $\mathrm{m}^{-3}$ ) plot for a $500 \mathrm{~nm}$ radius droplet hit by a laser pulse of intensity $I=10^{20} \mathrm{~W} / \mathrm{cm}^{2}$ at times (a) $3 \mathrm{fs}$, (b) $8 \mathrm{fs}$, (c) $16 \mathrm{fs}$, respectively. Angles are measured considering the center of the most dense region of the bunch. In the density scale, the maximum value corresponds to the critical density $1.8 \times 10^{27} \mathrm{~m}^{-3}$. The maximum absolute value of the electric field is $2.5 \times 10^{13} \mathrm{~V} / \mathrm{m}$. 
TABLE I. Table of electron bunches charge properties as inferred from simulations, for the smallest $(R=100 \mathrm{~nm})$ and the biggest $(R=1 \mu \mathrm{m})$ droplet, compared to the performances of other known attosecond bunch generation schemes. Apart from well-known ponderomotive acceleration, quite recent schemes such as REM and IFEL have been considered. The first consists of irradiating normally a solid nanofilm with an ultraintense femtosecond laser pulse, thus accelerating all the electrons in the laser spot simultaneously. The latter is an inverse-free-electron laser process, which can be coupled to a magnetic chicane in order to convert energy modulation into an optically spaced microbunch structure. In this way, stable attosecond electron bunches trains are formed.

\begin{tabular}{|c|c|c|}
\hline Droplet radius & Laser Irradiance & Predicted total charge \\
\hline $100 \mathrm{~nm}$ & $10^{19} \mathrm{~W} / \mathrm{cm}^{2}$ & $\approx 13.9 \mathrm{pC}$ \\
\hline $100 \mathrm{~nm}$ & $10^{20} \mathrm{~W} / \mathrm{cm}^{2}$ & $\approx 16.8 \mathrm{pC}$ \\
\hline $100 \mathrm{~nm}$ & $10^{21} \mathrm{~W} / \mathrm{cm}^{2}$ & $\approx 28 \mathrm{pC}$ \\
\hline $1 \mu \mathrm{m}$ & $1 \times 10^{19} \mathrm{~W} / \mathrm{cm}^{2}$ & $\approx 0.6 \mathrm{pC}$ \\
\hline $1 \mu \mathrm{m}$ & $10^{20} \mathrm{~W} / \mathrm{cm}^{2}$ & $\approx 0.9 \mathrm{pC}$ \\
\hline $1 \mu \mathrm{m}$ & $10^{21} \mathrm{~W} / \mathrm{cm}^{2}$ & $\approx 7.7 \mathrm{pC}$ \\
\hline Attosecond bunches production scheme & Total yielded charge & \\
\hline ponderomotive acceleration [1] & $<1 \mathrm{pC}$ & \\
\hline REM single bunch [4] & $>10 \mathrm{nC}$ & \\
\hline IFEL microbunches [5] & $\approx 1 \mathrm{pC}$ & \\
\hline
\end{tabular}

pulse may also counteract space-charge forces causing the bunches to spread in the far field, but we defer a detailed study to determine the maximum sustainable bunch charge for future work.

\section{CONCLUSIONS}

The emission mechanism of attosecond electron bunches from nanometer-sized droplets illuminated by a two-cycle laser pulse has been extensively studied, revealing a regime in which relativistic dynamics prevails over Mie optics in determining the final angular distribution of the bunches. The relevant condition $R \sim \delta_{r}=c / \omega_{p}^{r}$ is satisfied for sufficiently small droplet sizes and relativistic intensities $\left(I>10^{19} \mathrm{~W} / \mathrm{cm}^{2}\right)$, where induced transparency modifies the plasma frequency according to the relationship $\omega_{p}^{r}=\omega_{p} / \sqrt{\gamma}$, thus reducing the effective droplet extension to a few plasma skin depths. Under such conditions, the approximation for the existence of a proper bulk surface between the sphere and the medium is no longer valid and relativistic phenomena dominate through a combination of ponderomotive scattering followed by a subsequent bunch deviations as soon as they escape in vacuum. While the electrons run in phase with the laser pulse, space-charge forces and ponderomotive pressure combine to influence the final shape and traveling direction of the overdense bunches $\left(n_{b}>n_{c r}\right)$, which can reach very promising energies compared to the analogous ones produced by bigger droplets exhibiting a Mie-like behavior. In addition, they have a well-defined angular and energy distribution which can potentially be tuned for use in diverse applications such as generation of short-wavelength radiation and plasma diagnostics.

\section{ACKNOWLEDGMENTS}

The authors acknowledge computing resources under Grant No. JARA-HPC/JZAM04 at the Jülich Supercomputing Centre and thank Dr. H. Zilken and the visualization team of the Jülich Supercomputing Centre for assistance with the rendering for the 3D simulations. The EPOCH code used in this research was developed under UK Engineering and Physics Sciences Research Council Grants No. EP/G054940/1, No. EP/G055165/1, and No. EP/G056803/1.

[1] G. V. Stupakov and M. S. Zolotorev, Phys. Rev. Lett. 86, 5274 (2001).

[2] N. Naumova, I. Solokov, J. Nees, A. Maxsimchuk, V. Yanovsky, and G. Mourou, Phys. Rev. Lett. 93, 195003 (2004).

[3] I. Y. Dodin and N. J. Fisch, Phys. Rev. Lett. 98, 234801 (2007).

[4] V. V. Kulagin, V. A. Cherepenin, M. S. Hur, and H. Suk, Phys. Rev. Lett. 99, 124801 (2007).

[5] C. M. S. Sears, E. Colby, R. Ischebeck, C. McGuinness, J. Nelson, R. Noble, R. H. Siemann, J. Spencer, D. Walz, T. Plettner, and R. L. Byer, Phys. Rev ST Accel. Beams 11, 061301 (2008).

[6] T. V. Liseykina, S. Pirner, and D. Bauer, Phys. Rev. Lett. 104, 095002 (2010).

[7] H. C. Wu and J. Meyer-Ter-Vehn, Phys. Rev. Lett. 104, 234801 (2010).

[8] M. J. H. Luttikhov, A. G. Kachatryan, F. A. van Goor, and K. J. Boller, Phys. Rev. Lett. 105,124801 (2010),

[9] F. Y. Li, Z. M. Sheng, Y. Liu, J. Meyer-Ter-Vehn, W. B. Mori, W. Lu, and J. Zhang, Phys. Rev. Lett. 110, 135002 (2013). 
[10] T. Ditmire, T. Donnelly, A. M. Rubenchick, R. W. Falcone, and M. D. Perry, Phys. Rev. A 53, 3379 (1996).

[11] V. P. Krainov and M. B. Smirnov, Phys. Rep. 370, 237 (2002).

[12] U. Saalmann, Ch. Siedschlag, and J. M. Rost, J. Phys. B 39, R39 (2006).

[13] S. Sakabe, K. Nishihara, N. Nakashima, J. Kou, S. Shimizu, V. Zhakhovskii, H. Amitani, and F. Sato, Phys. Rev. A 69, 023203 (2004).

[14] Th. Fennel, K.-H. Meiwes-Broer, J. Tiggesbäumker, P.-G. Reinhard, P. M. Dinh, and E. Suraud, Rev. Mod. Phys. 82, 1793 (2010).

[15] T. V. Liseykina and D. Bauer, Phys. Rev. Lett. 110, 145003 (2013).

[16] J. Schreiber and L. Veisz (private communication).

[17] S. Zherebtsov, T. Fennel, J. Plenge et al., Nat. Phys. 7, 656 (2011).
[18] Extendable PIC Open Collaboration project UK and http:// ccpforge.cse.rl.ac.uk/gf/project/epoch/.

[19] C.F. Bohren and D. R. Huffmann, Absorption and Scattering of Light by Small Particles (Wiley, New York, 1983).

[20] P. W. Barber and S. C. Hill, Light Scattering by Particles: Computational Methods (World Scientific, Singapore, 1990).

[21] F. V. Hartemann, S. N. Fochs, G. P. Le Sage, N. C. Luhmann, Jr., J. G. Woodworth, M. D. Perry, Y. J. Chen, and A. K. Kerman, Phys. Rev. E 51, 4833 (1995).

[22] P. Gibbon, Short Pulse Laser Interactions with Matter (Imperial College Press, 2005), Chap. 5, p. 198.

[23] D. D. Meyerhofer, IEEE J. Quantum Elec. 33, 1935 (1997).

[24] G. Malka and J. L. Miquel, Phys. Rev. Lett. 77, 75 (1996).

[25] A. A. Andreev and K. Y. Platonov, Opt. Spectrosc. 114, 788 (2013). 